Results A total of 2645 women took part. 117 women reported childhood sexual abuse before age 15 years, a prevalence of $6.0 \%$ ( $95 \%$ CI $0.05 \%$ to $0.07 \%$ ). The abuse occurred mainly between ages 12 and 15 years. The majority of violence abusers were aged $30-39$ years and were male in $94.5 \%$ of the cases. Family members were responsible for the majority of abuse $(43.6 \%$, family friends $12.4 \%$, strangers $10.2 \%$ and other persons $10.2 \%$ ). A first occurrence of sexual occurrence age $<15$ years was reported by 203 women, of which $60.1 \%$ declared that it was forced $(p<0.001)$. A higher percentage $(64.7 \%)$ of women were abused once or twice, compared to few times $(18.7 \%)$ and many times $(16.6 \%)$. When the father was the perpetrator, the violence occurred many a few or many times. Conclusions The prevalence of child sexual abuse among women is considerable in Brazil. The participation of family members and the severity of the violence is notable. Patterns of abuse by the father suggest recurrent and regular abuse. Further studies about factors associated with this issue are needed.

\section{P2-503 FACTORS ASSOCIATED WITH CHILD SEXUAL ABUSE IN BRAZIL}

doi:10.1136/jech.2011.142976m.30

G B de Castro, ${ }^{*}$ I F Junior, L B Schraiber. Faculdade de Saúde Pública da Universidade de São Paulo, São Paulo, São Paulo, Southeastern, Brazil

Objective To analyse factors associated with child sexual abuse in Brazil.

Methods A cross-sectional study based on data of a multi-centre study performed by WHO. It used cluster sampling, with a representative sample of 1172 women aged 15-49 years in São Paulo, Southeastern Brazil, and 1473 women from Zona da Mata de Pernambuco, Northeastern Brazil. Standardised questionnaires were completed in 2000 and 2001. The follow variables (prior to the occurrence of sexual abuse) were analysed: sociodemographic characteristics (origin, migration) and family experiences of violence (mother abused by intimate partner, witness of violence in childhood). Non-conditional logistic regression was performed, with the estimative of ORs.

Results Physical violence by an intimate partner against the mother was associated with child sexual abuse in São Paulo $(\mathrm{OR}=2.37,95 \%$ CI 1.35 to 4.17 ) and in Zona da Mata de Pernambuco ( $O R=2.42$, $95 \%$ CI 1.38 to 4.23 ). Witnessing this violence was not associated with the outcome in both regions. Neither were sociodemographic characteristics.

Conclusions Girls exposed to violence against their mothers in their homes are in higher risk of suffering sexual abuse, regardless of some socio-demographic conditions. Studies on factors associated with abuse should be considered in the development of preventive policies and ensure children's rights.

\section{P2-504 ABORTION AND SEXUAL VIOLENCE: THE VULNERABILITY'S CONTEXT AMONG YOUTH WOMEN}

doi:10.1136/jech.2011.142976m.31

F B Pilecco, ${ }^{*}$ D R Knauth, Á Vigo. Universidade Federal do Rio Grande do Sul, Porto Alegre, Rio Grande do Sul, Brazil

Introduction Illegality of abortion perpetuates socioeconomic inequities in what it is practiced and, like sexual coercion, obscures its consequences for the women's health.

Purpose To investigate the relationship between abortion and experiences of sexual coercion among young women.
Methodology Data analysed come from GRAVAD research, a crosssectional study with stratified random sample, that was conducted with youth aged 18-24 years in Rio de Janeiro, Porto Alegre and Salvador. The present sample was composed by 870 interviews of women who reported having become pregnant. For statistical analysis was used Poisson regression with robust variance, incorporating structure of design and sample weights.

Results Abortion's statement was associated with account of one experience of sexual coercion ( $\mathrm{PR}=1.60,95 \% \mathrm{CI} 1.04$ to 2.44$)$. It has also appeared associated with higher education ( $\mathrm{PR}=2.78,95 \% \mathrm{CI}$ 1.77 to 4.36 ), greater number of sexual partners ( $\mathrm{PR}=2.21,95 \% \mathrm{CI}$ 1.31 to 3.75 ), not obtained the first information about sex with parents ( $\mathrm{PR}=1.90,95 \% \mathrm{CI} 1.14$ to 3.18$)$ and with a bigger number of pregnancies ( $\mathrm{PR}=1.65,95 \%$ CI 1.40 to 1.94 ) and sexual partners (PR $2.21 ; 95 \%$ CI 1.31 to 3.75 ). The prevalence of sexual coercion among young women in this study was $22.8 \%$.

Conclusion Association between abortion and sexual coercion reflects a situation of gender's vulnerability. Sexual violence appeared together with other factors, such as abortion, denoting precarious negotiations in terms of sexuality and reproduction. The silencing of sexual coercion by public institutions contribute to increased vulnerability of young people, as it blocks the access to educational, legal and health features, that could help breaking situations of coercion and to prevent it recurrence.

\section{P2-505 PREVALENCE AND RISK FACTORS ASSOCIATED WITH INFECTION BY LEISHMANIA INFANTUM IN DOGS FROM AN URBAN AREA OF BRAZIL IDENTIFIED BY MOLECULAR METHODS}

doi:10.1136/jech.2011.142976m.32

${ }^{1} \mathrm{~W}$ Coura-Vital, ${ }^{1,2} \mathrm{M}$ J Marques, ${ }^{1,3} \mathrm{~V} \mathrm{M}$ Veloso, ${ }^{1,3} \mathrm{~L}$ E Reis, ${ }^{1,3} \mathrm{~S}$ L Braga, ${ }^{1} \mathrm{M}$ H Morais, ${ }^{1,3}$ A Reis, ${ }^{1} \mathrm{M}$ Carneiro. ${ }^{*}{ }^{1}$ Universidade Federal de Minas Gerais, Belo Horizonte, Brazil; ' Universidade Federal de Alfenas, Alfenas, Brazil; ${ }^{3}$ Universidade Federal de Ouro Preto, Ouro Preto, Brazil

Introduction Over the last decades, visceral leishmaniasis (VL) has spread to a number of metropolitan areas in Brazil. The aim was to determine the prevalence and the risk factors associated with Leishmania infantum infection among seronegative and molecular positive dogs in an area of active transmission in Southeast of Brazil. Methods A cross-sectional study was conducted with 1443 dogs, random selected. Molecular methods were performed using polymerase chain reaction (PCR-RFLP) and serology was conducted using ELISA. Mixed logistic regression model was used to evaluate the risk factors associated with $L$ infantum infection.

Principal findings Of the 1443 dogs examined, 230 (15.9\%) were seropositive in ELISA assay, while PCR-RFLP revealed that 356 animals (24.7\%) were positive and 1087 (75.3\%) negative for $L$ infantum DNA. To identify the risk factors associated with infection, only seronegative animals $(n=1213)$ were evaluated, and of these 296 (24.4\%) were PCR-positive and 917 (75.6\%) PCR-negative for $L$ infantum DNA. The risk factors associated with infection were family income < one minimum salary (OR.4.1; 95\% CI 1.0 to 17.5), knowledge of the owner regarding the vector (OR.1.9; 95\% CI 1.1 to 3.4), permanence of dog in the backyard (OR.2.3; $95 \%$ CI 1.2 to 4.4), and lack of examination for VL (OR.1.6; 95\% CI.1.0 to 2.4 ).

Conclusions PCR is more accurate than serology in the early detection of infection among asymptomatic dogs, and its application on a large scale for the surveillance of VL could minimise false-negative results. Identification of the risk factors associated with canine visceral leishmaniasis is important for the definition of strategies of disease control. 